\title{
Non-variable cosmologically distant gamma-ray emitters as a propagation imprint of ultra-high-energy protons
}

\author{
A. Yu. Prosekin ${ }^{1}$, S. R. Kelner ${ }^{1}$, and F. A. Aharonian ${ }^{1,2}$ \\ 1 Max-Planck-Institut für Kernphysik, Saupfercheckweg 1, 69117 Heidelberg, Germany \\ e-mail: anton.prosekin@mpi-hd.mpg.de \\ 2 Dublin Institute for Advanced Studies, 31 Fitzwilliam Place, Dublin 2, Ireland \\ Received 10 May 2011 / Accepted 20 September 2011
}

ABSTRACT

\begin{abstract}
The acceleration sites of ultra-high-energy (UHE) protons can be traced by the footprint left by these particles when they propagate through cosmic microwave background (CMB) radiation. Secondary electrons produced in the extended region of several tens of Mpc emit their energy via synchrotron radiation predominantly in the initial direction of the parent protons. This forms a non-variable and compact (almost point-like) source of high-energy gamma rays. The importance of this effect is increased for cosmologically distant objects; because of severe energy losses, UHE protons cannot reach us even in the case of extremely weak intergalactic magnetic fields. Moreover, at high redshifts the energy conversion from protons to secondary particles becomes significantly more effective because of the denser and more energetic CMB in the past. This increases the chances of UHE cosmic rays to be traced by the secondary synchrotron gamma radiation. We discuss the energy budget and the redshift dependence of the energy transfer efficiency from UHE protons to synchrotron radiation. The angular and spectral distributions of radiation in the gamma- and X-ray energy bands are calculated and discussed in the context of their detectability by the Fermi LAT and Chandra observatories.
\end{abstract}

Key words. magnetic fields - X-rays: general - gamma rays: general - astroparticle physics

\section{Introduction}

Although there is a little doubt that ultra-high-energy (UHE) cosmic rays, $E \geq 10^{19} \mathrm{eV}$, are produced in extragalactic sources, the nature and origin of these objects remain unknown. In the case of very weak intergalactic magnetic fields (IGMF), the initial directions of these particles are only moderately entangled, and thus the information about their acceleration sites can be partly preserved. The quantitative treatment of the UHE cosmic ray propagation is limited because of large (orders of magnitude) uncertainties of the relevant model parameters. This especially concerns the IGMF. The current measurements yield only an upper limit of $B \sim 10^{-9} \mathrm{G}$ for the component parallel to the line of sight (Ryu et al. 1998; Blasi \& Olinto 1999). The lower bound is estimated from the non-observations of GeV gamma-ray counterparts from distant $\mathrm{TeV}$ blazars. Depending on the assumptions concerning the cascade development, its suppression, and the upper limit on the variability timescale of the TeV gamma-ray emission, the values of the lower limit inferred by different authors vary from $B \sim 10^{-16}-10^{-15} \mathrm{G}$ (Neronov \& Vovk 2010; Dolag et al. 2011; Tavecchio et al. 2010) to $B \sim 10^{-18} \mathrm{G}$ (Dermer et al. 2011). The reasons for this broad range of IGMF estimates have been critically discussed by Taylor et al. (2011). The largescale structure of the IGMF is also very uncertain; the attempts to model usually lead to quite different results (Dolag et al. 2005; Sigl et al. 2004). These uncertainties significantly limit the potential of the so-called cosmic-ray astronomy. Moreover, even in the case of very weak magnetic fields, the cosmic ray "horizon" of UHE cosmic ray sources through direct detection of these particles is limited by interactions with the cosmic microwave background radiation (CMBR). For the highest energy particles, $E \sim 10^{20} \mathrm{eV}$, it does not exceed $100 \mathrm{Mpc}$. At lower energies cosmic rays can propagate to significantly longer distances, but they are dramatically deflected by galactic magnetic fields. These two factors significantly limit the potential of the so-called proton astronomy.

An important information about the acceleration sites of UHE protons is contained in gamma rays produced during the propagation of protons through the CMBR. For a very low intergalactic magnetic field the secondary products of $p \gamma$ interactions initiate electromagnetic cascades in the CMBR. The effective development of these cascades is determined by the dominance of Compton energy losses over the synchrotron losses of secondary electrons. Generally, this condition is quite relaxed; it requires an IGMF weaker than $10^{-10} \mathrm{G}$. However, the second condition of the detection of cascade gamma rays in the direction of parent UHE protons requires much weaker magnetic fields, less than $10^{-15} \mathrm{G}$. Otherwise the relatively low energy electrons, and consequently the $\mathrm{GeV}$ and $\mathrm{TeV}$ gamma-ray emission, will be immediately isotropised. Formally, these weak intergalactic magnetic fields cannot be excluded. Moreover, it has been argued recently that very high-energy tails of gamma-ray spectra of distant $\mathrm{TeV}$ blazars might be contributed by cascades initiated by UHE protons in the intergalactic medium with a magnetic field as weak as $10^{-15} \mathrm{G}$ (Essey et al. 2011).

Remarkably, even for a strong IGMF we might expect physically extended (but looking point-like) gamma-ray sources formed around production sites of UHE cosmic rays (Aharonian 2002; Gabici \& Aharonian 2005; Aharonian et al. 2010; Kotera et al. 2011). In this case gamma rays are produced through synchrotron radiation of secondary electrons from photomeson interactions. Protons lose a significant fraction of their energy on scales of several tens of Mpc. If the magnetic field in these regions located at relatively low redshifts, $z \ll 1$, is sufficiently 
strong, $B>10^{-10} \mathrm{G}$ (so the secondary electrons radiate their energy predominantly via synchrotron process), but weaker than $10^{-7} \mathrm{G}$ (so that the initial directions of electrons are not dramatically changed), the resulting gamma-ray synchrotron sources can be quite compact with an angular size as small as $0.1^{\circ}$.

For cosmologically distant sources embedded in denser $\left(n_{\mathrm{ph}} \propto(1+z)^{3}\right)$ and more energetic $(T \propto 1+z)$ CMBR, protons lose their energy on distances significantly shorter than $100 \mathrm{Mpc}$. For a magnetic field of strength $B=10^{-10}-10^{-8} \mathrm{G}$ the high-energy electrons produced in photomeson processes intensively emit synchrotron radiation in the $\mathrm{GeV}$ range of energies. The electrons radiate most of their energy on the almost rectilinear part of the path that gives, along with the weak deflection of protons, a collimated beam of gamma rays that points to the acceleration site of UHE protons. Note that the considerable contribution to the high-energy electrons is provided by the gamma rays produced in decay of mesons. The interaction of these gamma rays with cosmic radio background radiation occurs in the regime when most of the energy goes to one of the components of the created electron-positron pair.

The probability of detecting cosmic ray sources via synchrotron radiation of secondary electrons strongly depends on the maximum energy of accelerated protons. For sources with redshift $z \ll 1$, the interaction of protons with the CMBR is effective only when the proton spectrum extends to $10^{20} \mathrm{eV}$ and beyond. Because an acceleration of protons to these high energies can be realized only for a unique combination of a few principal parameters, in particular the linear size of the source, the strength of the magnetic field, and the Lorentz factor of the the bulk motion (Aharonian et al. 2002), the number of sources of $10^{20} \mathrm{eV}$ cosmic rays can be quite limited compared to sources that accelerate particles to $E_{\max } \sim 10^{19} \mathrm{eV}$. Because protons at high redshifts interact with denser and more energetic photons of the CMBR, the requirement to $E_{\max }$ is significantly relaxed, consequently one should expect a dramatic increase of the number of UHE cosmic ray sources surrounded by gamma-ray halos. Furthermore, at high redshifts the interaction of protons with the CMBR via the pair production (Berhe-Heitler) process begins to play an important role and a considerable part of the proton energy is converted to less energetic electrons compared to the electrons generated in photomeson reactions. Appearance of dense halos of Bethe-Heitler electrons around the source at presence of magnetic field of the order of $B \sim 10^{-6} \mathrm{G}$ (comparable to the field of clusters of galaxies) results in radiation dominated by synchrotron X-rays.

In this paper we study the energy and angular distribution of the synchrotron gamma-ray emission from cosmologically distant sources. The calculations are based on the approach developed in our previous work (Aharonian et al. 2010) combined with cosmological effects on the propagation of gamma rays and protons. The space in the vicinity of the source, where all relevant processes occur, can be considered as a conventional one. Therefore, taking into account denser ambient radiation at high redshifts, we can apply the developed formalism to calculate the distribution function of gamma rays in the vicinity of the source. Using this function, we can easily obtain the distribution function of the observed radiation, which has propagated cosmological distance (see Appendix A).

\section{Energy budget}

An accelerator of UHE protons located at high redshift has appreciably different conditions of ambient media as compared to those in the nearby Universe. The photon field of the CMBR is

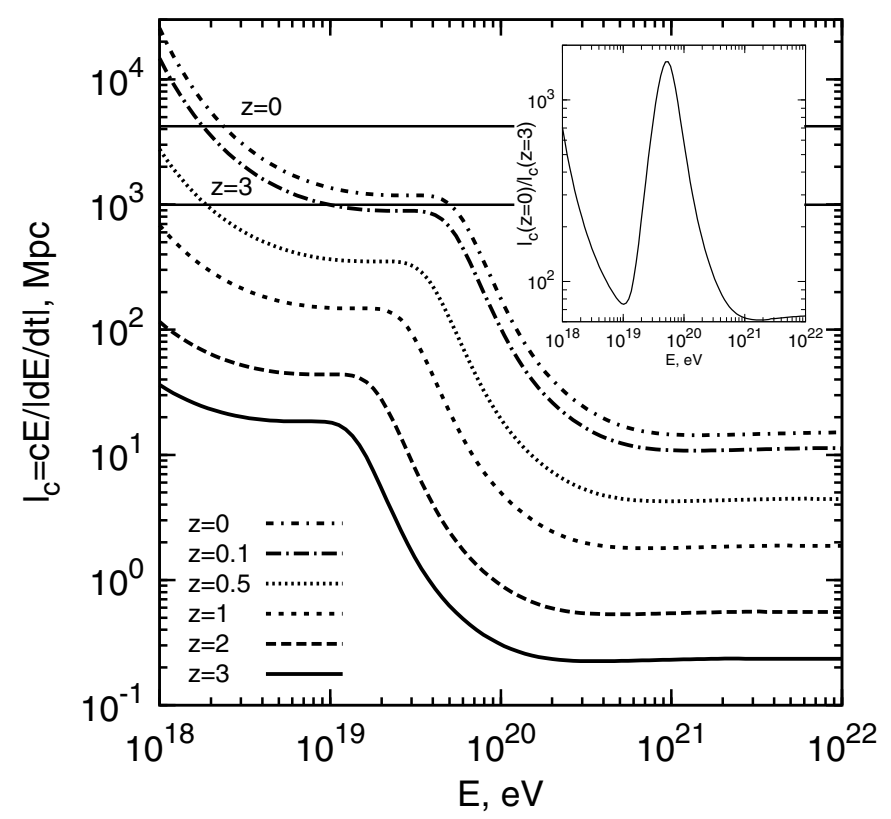

Fig. 1. Cooling length of protons in the intergalactic medium through interactions with photons of the CMBR at different redshift. The ratio of cooling lengths at $z=0$ and $z=3$ are shown in the inset. The horizontal lines correspond to adiabatic energy losses caused by the expansion of Universe at redshifts $z=0$ and $z=3$.

denser and more energetic because of cosmological expansion. The increase of density by a factor of $(1+z)^{3}$ leads to more frequent interactions of UHE protons with the CMBR, which in turn intensifies the energy loss rate. The average energy of photons is also increased by a factor of $(1+z)$, which decreases the threshold energy of the interactions for protons. The energy loss rate of protons through interaction with the CMBR at the epoch of redshift $z$ is expressed in terms of the present loss rate $\beta(E)=-\frac{1}{E} \frac{\mathrm{d} E}{\mathrm{~d} t}$ as

$\beta(E, z)=(1+z)^{3} \beta_{0}((1+z) E)$.

It is convenient to present this relation in terms of the cooling length which is shown in Fig. 1. The cubic dependence on redshift leads to a considerable decrease of the cooling length. In particular, for the constant losses at highest energies, $E \geq$ $10^{21} \mathrm{eV}$, it is reduced from $\approx 15 \mathrm{Mpc}$ at the present epoch to $\approx 0.2 \mathrm{Mpc}$ at $z=3$. The plateau of constant losses itself extends to lower energies because of the energy shift. At lower energies the combination of the effects related to the energy shift and the increase of density jointly results in a reduction of the cooling length by a factor higher than $(1+z)^{3}$. Indeed, as the inset in Fig. 1 shows, the reduction of the proton cooling length can be an order of magnitude higher. The peaks show domains where energy loss at $z=3$ is the most intensive with regard to the present epoch. If the energy cutoff in the initial proton spectrum falls into this domain, the advantage of energy extraction at cosmological distances becomes more significant. Moreover, at $E=10^{18} \mathrm{eV}$, the cooling length is reduced from $\geq 10^{4} \mathrm{Mpc}$ at $z=0$ to tens of $\mathrm{Mpc}$ at $z=3$. It is interesting to note that the energy loss rate of protons of energy $E<10^{19} \mathrm{eV}$ at $z=3$ is comparable to the loss rate of $E \geq 10^{20} \mathrm{eV}$ protons at the present epoch.

Figure 2 describes the evolution of proton energy losses and the efficiency of their conversion to the electron component of secondaries with the distance to the source at different redshifts. The electron component includes the electrons produced through 

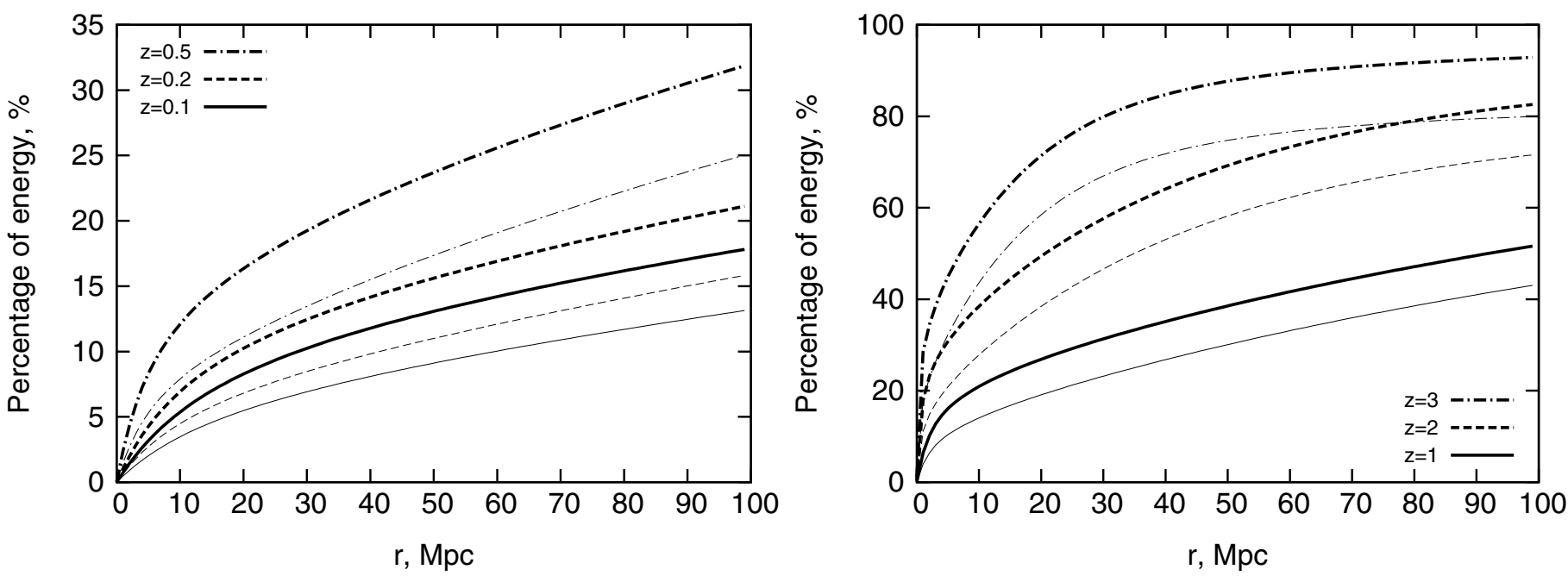

Fig. 2. Fraction of the initial energy of protons with $E>10^{18} \mathrm{eV}$ lost (thick lines) converted into the energy of electrons (thin lines) at the distance $r$ from the source. The injected proton spectrum is assumed a power-law with an exponential cutoff $J_{\mathrm{p}}(E)=J_{0} E^{-2} \exp \left(-E / E_{0}\right)$, with $E_{0}=3 \times 10^{20} \mathrm{eV}$.

all channels under consideration: pair production by protons, decay of charged mesons produced in photomeson processes and pair production by gamma rays produced from the decay of neutral mesons. As mentioned above, in the latter process almost all energy of the photon goes to the energy of one of the electrons. Therefore the gamma rays can be treated as electrons. Then the energy removed by neutrinos is the difference between the energy lost by protons and the energy converted to electrons. As can be seen from Fig. 2, the protons with initial energies $E>10^{18}$ lose only $18 \%$ of their total energy after passing $r=100 \mathrm{Mpc}$ at the redshift $z=0.1$, whereas at redshift $z=3$ the same protons lose $93 \%$ of the available energy already at $r=70 \mathrm{Mpc}$, where the saturation begins. This is because the cooling length of protons in the range $E>10^{18} \mathrm{eV}$ does not exceed tens of Mpc at $z=3$, while for redshifts $z \ll 1$, the protons have a cooling length of cosmological scales relative to the pair production process. As the contribution of pair production process increases, the part of the energy transferred from protons to electrons increases from $74 \%$ at $z=0.1$ to $86 \%$ at $z=3$.

The fraction of the initial energy of the protons with $E>10^{18} \mathrm{eV}$ that can be converted to the electrons generated at photomeson processes depends strongly on the position of the cutoff energy. Left panel of Fig. 3 shows that the extraction efficiency of the proton energy and its transfer to this component of electrons grows with the redshift, and at $z=3$ all energy available for conversion is transferred at first $5 \mathrm{Mpc}$. The fraction of proton energy taken away by neutrinos in photomeson processes is $42 \%$ independently of redshift. Owing to the more energetic photons of the CMBR, the threshold of photomeson interactions is shifted to lower energies, which leads to an increase of extracted energy from $6 \%$ to $17 \%$ (see Fig. 3). However, as pair production begins to play a significant role at high redshifts, the part of the electrons generated in photomeson processes decreases from $49 \%$ to $22 \%$ at a distance $r=100 \mathrm{Mpc}$.

The importance of the reaction threshold shift on the production of electrons becomes more obvious if the protons with energies close to the threshold and higher are taken into account. The percentage of initial energy of protons with energies $E>10^{19} \mathrm{eV}$, which is converted to the energy of electrons, is presented in the right panel of Fig. 3. While at $z=0.1$ the production of electrons through photomeson processes dominate at all distances shorter than $100 \mathrm{Mpc}$, at high redshifts the pair production prevails.

\section{Gamma-ray source}

Because they possess the highest energy among secondaries, the electrons generated in photomeson processes emit almost all their energy via synchrotron radiation. Therefore, because of the shift of the threshold of photomeson interactions, the intensity of synchrotron gamma rays increases with the redshift of the source. On the other hand, the reduction of cooling length of protons results in the reduction of the apparent angular size of the region that emits the synchrotron radiation, in addition to the decrease because of geometrical factor.

Figure 4 shows the spectral energy distribution (SED) of synchrotron gamma rays observed within different polar angles in the direction of the proton source. The calculations were performed for an IGMF with the strength of $B=10^{-9} \mathrm{G}$ and the coherence length that depends on redshift as $\lambda=1 /(1+z) \mathrm{Mpc}$. The geometry of the expanding Universe leads to a more complicated dependence of the apparent angular size on distance compared to the $\sim 1 / r$ dependence for the local Universe. It is useful to define a reference angle $\theta_{z}^{*}$ that is equal to the angular size of the region with the radius $r=0.5 \mathrm{Mpc}$ located at the redshift $z$. Expressing the angles in units of corresponding reference angles, we can compare the regions located at different redshifts by eliminating the geometrical factor. As expected, at high redshifts the reference angle increases with $z$ (see Appendix A, Fig. A.1). Figure 4 presents the fluxes for each redshift within three polar angles that differ from each other by a factor of 3 and are expressed in units of the corresponding reference angle. The maximum angle indicated in the plots is the polar angle within which the total flux is observed. Comparison of the maximal angles in units of reference angles shows a tendency of decreasing the angular size of the region of secondary synchrotron radiation with redshift from $9 \theta_{0.1}^{*}$ to $\theta_{3}^{*} / 9$.

The interaction of synchrotron gamma rays with extragalactic background light (EBL) leads to considerable attenuation of the flux. As Fig. 4 shows, the intergalactic absorption becomes substantial, depending on the distance to the source, from $\mathrm{TeV}$ energies down to tens of $\mathrm{GeV}$ energy range. To calculate the 

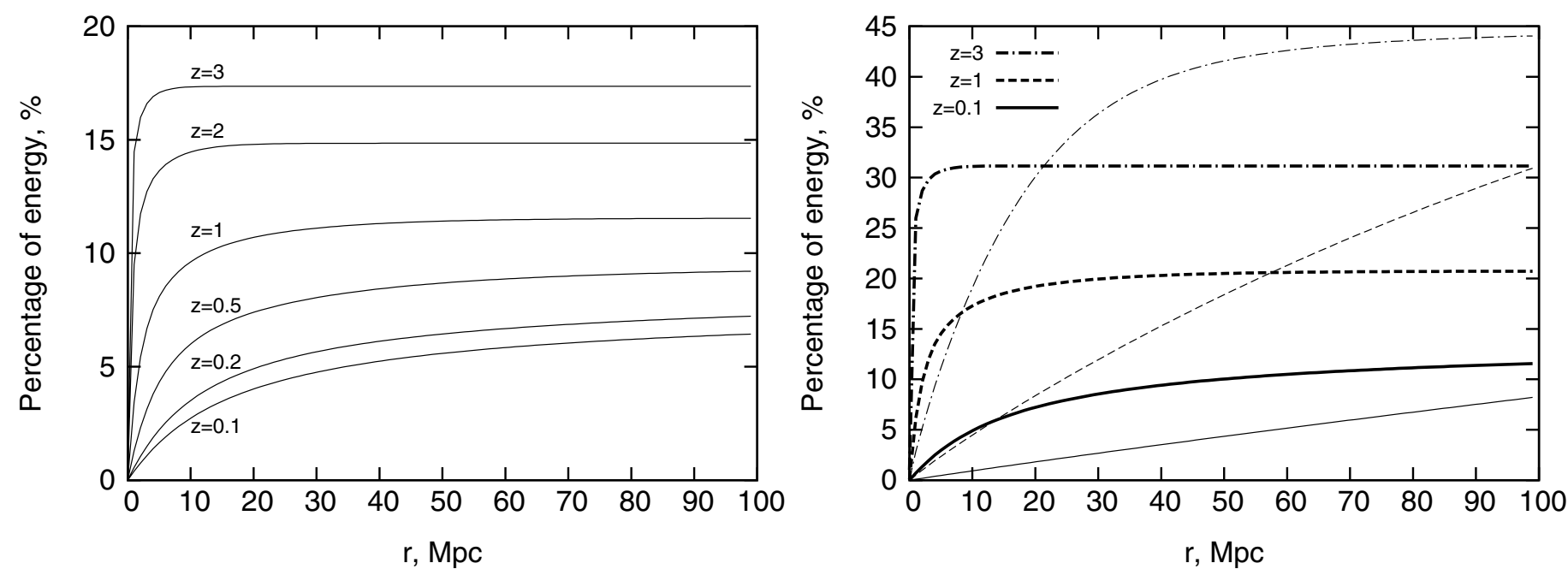

Fig. 3. Left panel: the fraction of the initial energy of protons with energy $E>10^{18} \mathrm{eV}$ converted to the energy of electrons from photomeson production at a distance $r$ from the source. Right panel: the percentage of the initial energy of protons with energy $E>10^{19} \mathrm{eV}$ converted to the energy of electrons from photomeson production (thick lines) and electrons from pair production (thin lines) at a distance $r$ from the source.
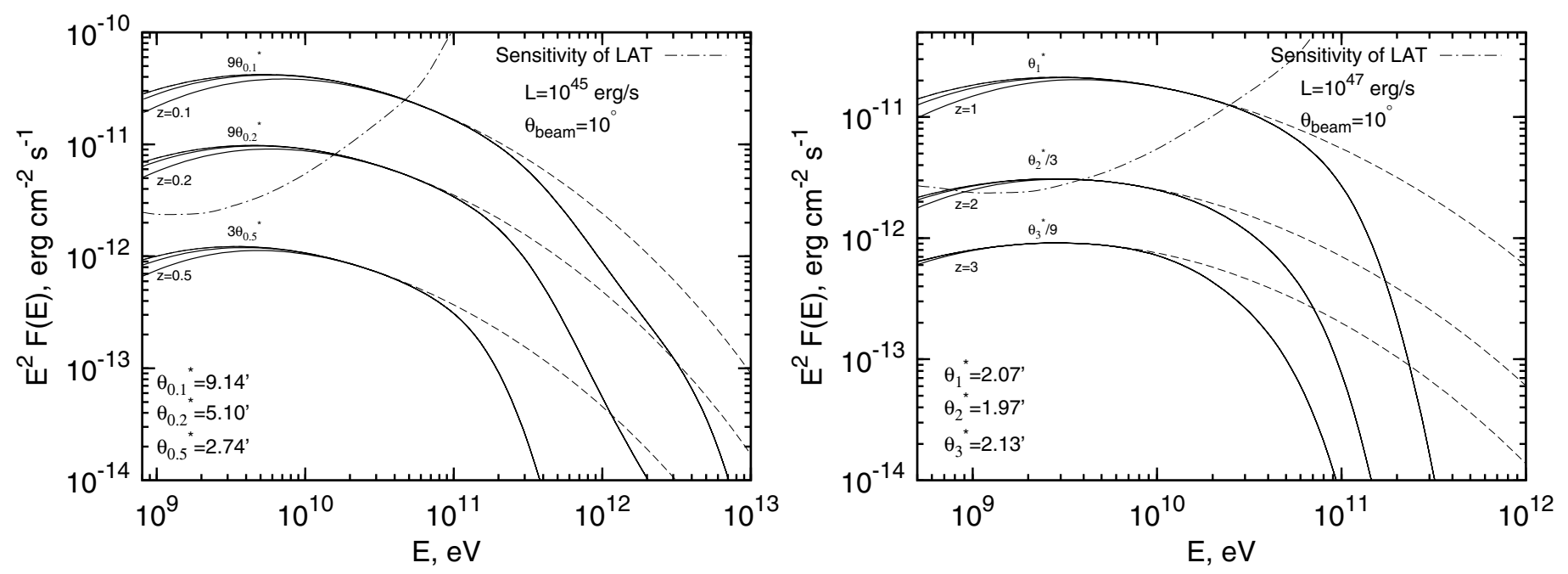

Fig. 4. Spectral energy distribution of gamma rays observed within different angles in the direction of the source with EBL absorbtion (thick lines) and without (dashed lines) indicated for maximum angles. $\theta_{z}^{*}$ is the apparent angular size of the region with radius $0.5 \mathrm{Mpc}$ from the distance with redshift $z$. The angles are specified in units of the corresponding $\theta_{z}^{*}$ and differ from each other by a factor of 3 . The maximum angles for every redshift, the total power of injection of protons $L$ and the beaming angle $\theta_{\text {beam }}$ are indicated. The IGMF is fixed at the strength of $B=1 \mathrm{nG}$, while the coherence length is taken as $\lambda=1 /(1+z) \mathrm{Mpc}$.

absorbtion of gamma rays, the model of the EBL developed in Franceschini et al. (2008) have been applied.

At high redshifts the electrons from photomeson reactions are produced close to the acceleration cites of protons. In this case a considerable part of the emitting electrons might be found in a much stronger magnetic field compared to the average intergalactic field. The energy spectrum of synchortron radiation of secondary electrons is shifted linearly with the change of the magnetic field strength to keep the ratio $E_{\gamma} / B$ constant (see Aharonian et al. 2010). Therefore the increase of the strength of magnetic field leads to the shift of radiation spectrum towards higher energies; if the radiation extends to TeV energies, the intergalactic absorption becomes quite violent; this results in the dissipation of almost the entire radiation. The absorbed gamma rays initiate cascades that contribute to the diffuse extragalactic background radiation.

Keeping in mind the sensitivity of instruments such as Fermi, the detection of the collimated synchrotron radiation from sources located at high redshifts is possible only in case of very powerful AGN. The anisotropic injection of UHE cosmic rays allows us to reduce the required power of the source. Until the deflection of protons is smaller than the angle of a jet, there is no difference between the spherically symmetric and anisotropic case. In both cases the observer will see identical pictures. For the given power of injection the existence of anisotropy with the opening angle $\theta_{\text {beam }}$ of the jet means increase of the gamma rays flux by a factor $4 / \theta_{\text {beam }}^{2}$ compared to the spherically symmetric case. The fluxes of synchrotron gamma rays in Fig. 4 are calculated for the case of $\theta_{\text {beam }}=10^{\circ}$. The deflection of protons is lower than this value of the opening angle while protons produce secondary particles. Figure 4 shows that at the power of the proton injection $L=10^{45}-10^{47} \mathrm{erg} \mathrm{s}^{-1}$, the fluxes of gamma rays can exceed the sensitivity (minimum detectable fluxes) of Fermi LAT (Atwood et al. 2009). The differential sensitivity indicated in Fig. 4 is for the $5 \sigma$ detection for one year of sky survey exposure. The resolution of the Fermi LAT 
varies depending on the photon energy from $42^{\prime}$ at $E=10^{9} \mathrm{eV}$ to $4.2^{\prime}$ at $E=10^{11} \mathrm{eV}$ (Atwood et al. 2009). Already for the source located at $z=0.2$ the angular size is $46^{\prime}$, consequently the source of gamma rays would be seen as an extended one only if $z<0.2$. Therefore, for the source located at $z=0.1$ the flux of energy between angles $3 \theta_{0.1}^{*}=27.42^{\prime}$ and $9 \theta_{0.1}^{*}=82.26^{\prime \prime}$ equals $3.6 \times 10^{-12} \mathrm{erg} \mathrm{s}^{-1} \mathrm{~cm}^{-2}$, which exceeds the sensitivity of the Fermi LAT. Therefore this part of radiation can be resolved and detected as a weak halo around the point source.

There are differences in the detection of synchrotron gammaray radiation and the radiation produced through an electromagnetic cascade. In an extremely weak IGMF the deflections of electrons during the last stages of the cascade become sufficiently small to result in a quite compact halo of gammaray radiation. Ando \& Kusenko (2010) have reported gammaray halos in the stacked images of AGNs with an angular size $0.5^{\circ}-0.8^{\circ}$ (see, however, Neronov et al. 2011), which is consistent with a strength of the IGMF $B \approx 10^{-15} \mathrm{G}$. The angular size of this halo is comparable with the angular size given by synchrotron gamma-ray radiation for the sources of UHE cosmic rays located at $z=0.1-0.2$ (see Fig. 4). However, the SEDs of the radiations produced through these two processes are different. The SED of the cascade radiation has its maximum at higher energies, and the slope before (after) maximum is flatter (steeper) than the slope of the SED of the synchrotron radiation (see, e.g., Taylor et al. 2011; Essey et al. 2011).

\section{X-ray emission}

Protons lose part of their energy via pair production. The pairproduced electrons have lower energies compared to the electrons produced in photomeson processes and emit synchrotron radiation in the lower band of the spectrum. At high redshifts the mean free path of protons relative to pair production process is decreased and generated electrons are located in a more compact region. Moreover, the fraction of the energy of the protons converted to the secondary electron component increases and reaches $68 \%$ at $z=3$ (see Figs. 2, 3).

Synchrotron radiation for a strength of the IGMF of $B=10^{-9} \mathrm{G}$ and inverse Compton (IC) scattering give an equal contribution to electron losses at energy $E \approx 3 \times 10^{18} \mathrm{eV}$. At lower energies the electrons lose their energy predominantly through IC scattering. This scattering happens in the KleinNishina regime and almost all energy is transferred to the photons. In turn the high-energy photons produce electron-positron pairs due to interaction with the CMBR and most of the energy is transferred to one component of the pair, which again suffer IC scattering. This process can be considered as an alternation of the particle state with a gradual reduction of energy. The electromagnetic cascade produces a huge halo of gamma rays with energy in $\mathrm{GeV}$ region. The region of the synchrotron radiation is more compact, and electrons radiate it at the place of their generation. Despite this, the region is still quite extended, as can be seen from angular distribution in Fig. 5. The flux of the synchrotron radiation drops at low energies and becomes very low in the X-ray region. The calculation of fluxes presented in Fig. 5 includes only a homogeneous IGMF with strength $B=10^{-9} \mathrm{G}$. However, the strength of magnetic fields close to the accelerator can be much higher. To take this into account, we considered the following spatial distribution for the IGMF magnetic field:

$B=B_{\mathrm{cl}}\left(\frac{r_{0}}{r+r_{0}}\right)^{3}+B_{0}$,

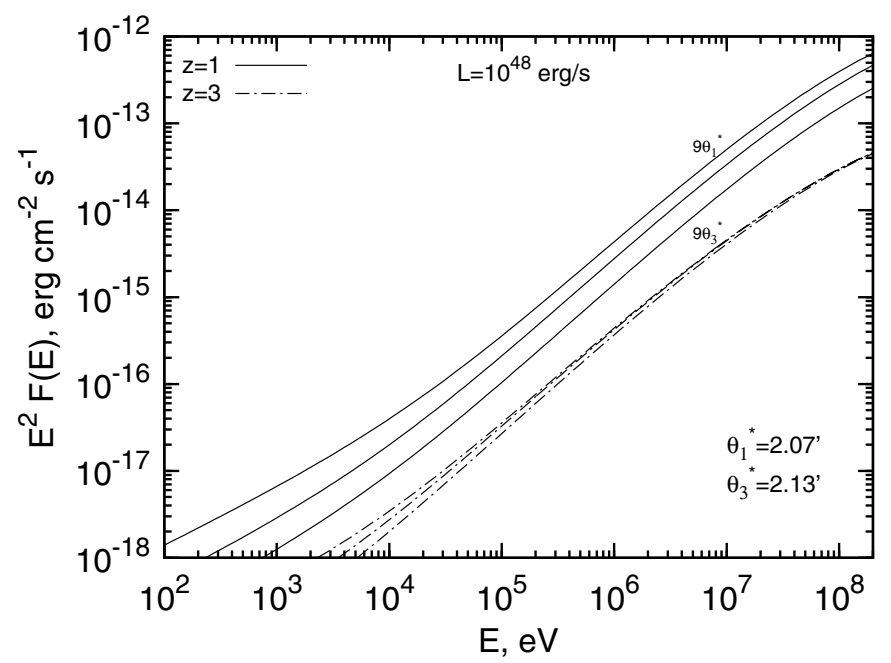

Fig. 5. Energy flux distribution of gamma- and X-rays observed within different angles in the direction of the source for the case of homogeneous magnetic field $B=1 \mathrm{nG}$. Other parameters are the same as in previous figures.

where $B_{\mathrm{cl}}=10^{-6} \mathrm{G}$ is the magnetic field in the cluster of galaxies with a characteristic size of $r_{0}=1 \mathrm{Mpc}, B_{0}=10^{-9} \mathrm{G}$ is the IGMF. This dependence of the magnetic field was chosen to produce a dipole behavior at long distances. For this case the energy and angular distributions of gamma- and X-rays are presented in the left panels of Figs. 6 and 7. This magnetic field increases considerably the fluxes of synchrotron radiation at low energies, which is generated in the small region close to the source and therefore has a narrow angular distribution. The right panels show that the contribution of electrons produced in a pairproduction process (lower lines) is dominant in the X-ray region, whereas gamma rays are generated predominantly by electrons produced in photomeson processes. The horizontal segments indicate the sensitivity of Chandra (Lehmer et al. 2005; Romano et al. 2008) for corresponding maximum angles of observation. Evidently, the low-energy part of X-rays $(0.5-2 \mathrm{keV})$ is detectable for a specified power of injection of protons, whereas the radiation at higher energies can be missing for sources located at high redshifts because Chandra is less sensitive in the range of energies $2-8 \mathrm{keV}$.

It follows from Figs. 6 and 7 that the electrons produced in photomeson processes provide the main contribution to synchrotron gamma rays, although most of the energy lost by protons is contained in the low-energy component of secondary electrons from the pair-production process. The radiation of this component can be detected only for a quite strong, $B_{\mathrm{cl}} \simeq 10^{-6} \mathrm{G}$ magnetic field around the source. This can happen if the source is located inside a cluster of galaxies with a typical size of $1 \mathrm{Mpc}$. Nevertheless, even in the case of intensive pair-production that takes place in cosmologically distant objects, only a small fraction of pairs is produced in a relatively compact region with a linear size of $\sim 1 \mathrm{Mpc}$ (see Fig. 2); most of proton energy is converted to the energy of the extended gamma-ray halo.

\section{Summary}

High-energy gamma rays are unavoidably formed around the sources of UHE cosmic rays because of the synchrotron radiation of secondary electrons produced at interactions of the highest energy protons with the cosmic microwave background radiation. In our previous paper (Aharonian et al. 2010) we 

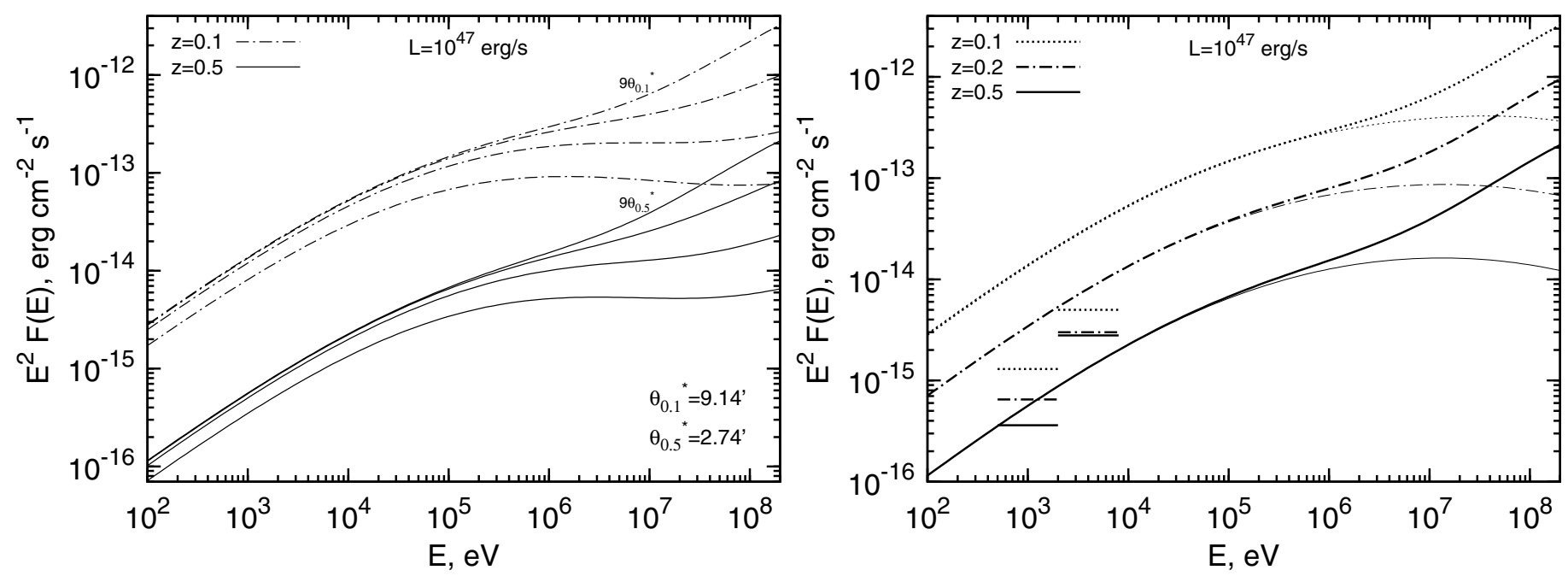

Fig. 6. Energy flux distribution of gamma- and X-rays observed within different angles in the direction of the source (left panel) and within maximum angle (right panel) for a two-band magnetic field. For the right panel, the total radiation from photomeson and pair production electrons (upper lines) and the radiation from pair production electrons (lower lines) are indicated. Horizontal segments present the Chandra sensitivity for the corresponding maximum angles of observation. The other parameters are the same as in previous figures.
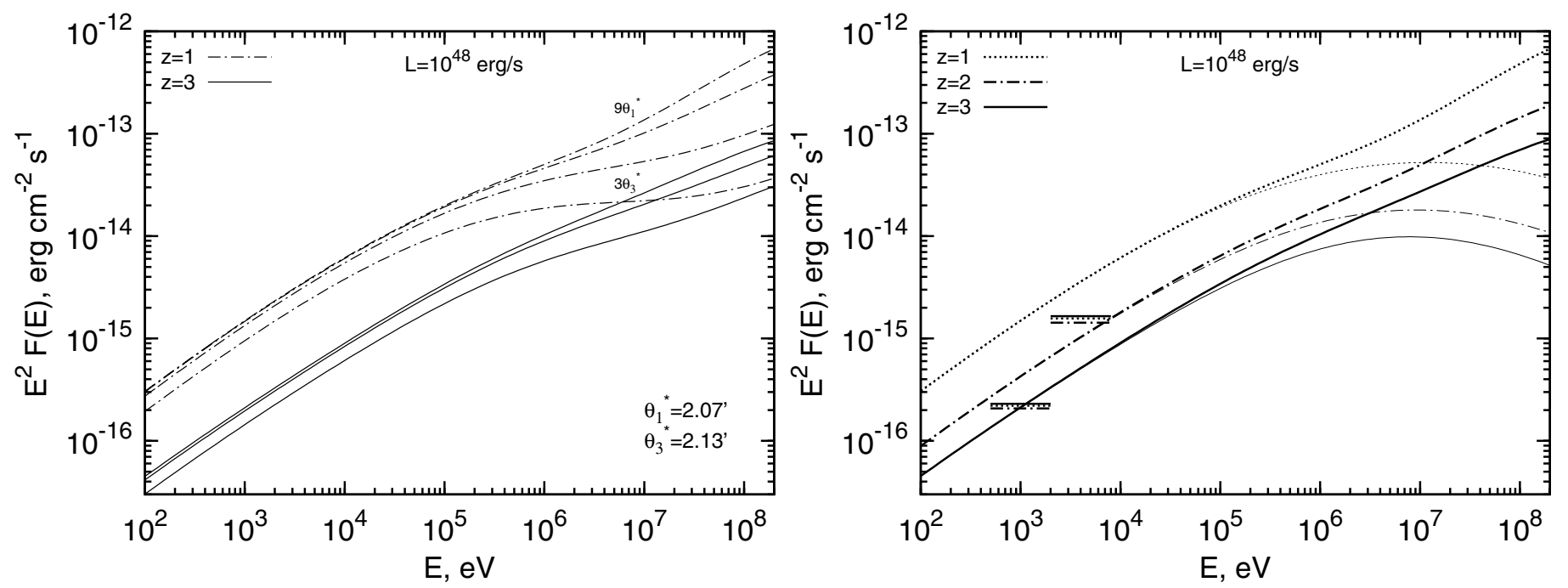

Fig. 7. Same as Fig. 6 but for $z=1,2,3$ and the total power of injection of protons $L=10^{48} \mathrm{erg} \mathrm{s}^{-1}$.

have shown that even for relatively strong intergalactic magnetic fields in the neighborhood of UHE cosmic ray accelerators, $B \sim 10^{-7}-10^{-9} \mathrm{G}$, these processes lead to the formation of high-energy point-like gamma-ray sources. Because of relatively weak gamma ray signals, the chances to detect these sources are higher for objects located in the nearby Universe, namely at distances less than $100 \mathrm{Mpc}$. Because the conversion efficiency of the proton energy to secondary gamma rays is dramatically reduced at proton energies $E \leq 10^{20} \mathrm{eV}$, one may hope to detect gamma rays only from extreme objects that accelerate protons to energies $10^{20} \mathrm{eV}$ and beyond. Given the fact that this requirement can be satisfied only for a unique combination of parameters and for the limited volume of the $\leq 100 \mathrm{Mpc}$ region, realistically one can expect only a small number of these sources. One can gain a lot if one extends the study to cosmological distances. First of all, this allows one to probe the most powerful objects in the Universe (e.g. quasars and AGNs) in which more favorable conditions can be formed for the acceleration of protons to energies $10^{20} \mathrm{eV}$. Secondly, because of higher temperature of the CMBR at cosmological epochs, i.e., because of denser and hotter relic photons, less energetic protons (with energies down to $10^{19} \mathrm{eV}$ ) can lead to an effective production of gamma rays. Given that the conditions of acceleration of protons to $10^{19} \mathrm{eV}$ in suspected cosmic accelerators are much more relaxed compared to the $10^{20} \mathrm{eV}$ extreme accelerators (see Aharonian et al. 2002), we should expect a dramatic increase of these gammaray sources. Another factor of enhancement for the number of sources comes from the increase of the volume of the explored region to redshifts $z \geq 3$. An obvious caveat in this case is the decrease of the gamma-ray flux. However, this factor can be at least partly compensated for by the huge power of objects in the remote Universe. Moreover, owing to dramatic reduction of mean free paths of UHE protons at cosmological epochs $z \geq 1$, the conversion efficiency of proton energy to gamma rays is increased by almost an order of magnitude, which makes these objects extremely effective gamma-ray emitters. Finally, because the secondary gamma-ray emission generally follows the direction of parent protons, beamed cosmic accelerators like GRBs 


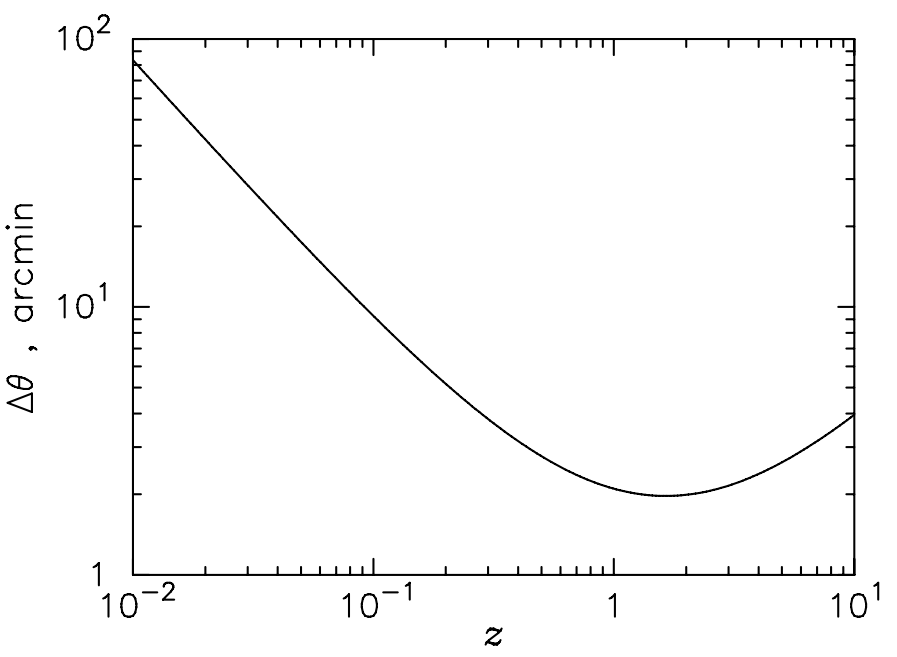

Fig. A.1. Angular size of the source with diameter $D=1 \mathrm{Mpc}$ located at different redshifts $z$.

and blazars seem to be quite attractive targets for the search of point-like but steady GeV gamma-ray emission from cosmologically distant objects.

\section{Appendix A: Angular size of sources at high redshifts and distribution function in the expanding Universe}

If the radiation is generated on scales smaller than cosmological ones, the relevant processes can be considered as they take place in the conventional stationary Euclidean space. However, when radiation propagates over cosmological distances, the expansion of space should be taken into account. Based on the law of free motion of massless particles in expanding space, one can find the distribution function of photons at the observation point. Then we need to convert the distribution function calculated in the reference frame with an origin at the source to the reference frame of the observer (Kelner et al. 2011). In the present paper the free propagation of photons is considered in the flat expanding Universe with parameters $\Omega_{\Lambda}=0.73, \Omega_{\mathrm{m}}=0.27$ and $H_{0}=71 \mathrm{~km} \mathrm{~s}^{-1} \mathrm{Mpc}$.

We consider an isotropic gamma-ray source of radius $R_{*}$ located at redshift $z$. Let us assume that photons escape this region with spherically symmetric distribution $f_{z}(E, \theta)$, where $E$ is the energy of photon and $\theta$ is the angle between photon momentum and radial direction at the escape point. Finally we assume that after the escape gamma rays propagate freely. In the case of small angles the distribution function $f_{0}(z, E, \Omega(\theta))$ of gamma rays at the observation point integrated over the solid angle $\Omega$ (with polar angle $\theta$ ) can be expressed in the following form:

$f_{0}(z, E, \Omega(\theta))=2 \pi\left(\frac{\Theta_{*}}{1+z}\right)^{2} \int_{0}^{\theta / \Theta_{*}} f_{z}(E(1+z), x) x \mathrm{~d} x$,

where

$\Theta_{*}=\left(\frac{c}{H_{0} R_{*}(1+z)} \int_{0}^{z} \frac{\mathrm{d} z^{\prime}}{\sqrt{\Omega_{\mathrm{m}}\left(1+z^{\prime}\right)^{3}+\Omega_{\Lambda}}}\right)^{-1}$.

The final result (A.1) differs from the analogous one corresponding to the stationary space by the dependence on the redshift as well as by the nonlinear dependence of the angular size on distance. The latter is determined by the parameter $\Theta_{*}$ given by Eq. (A.2). The parameter $\Delta \theta=2 \Theta_{*}$ has the meaning of the angular size of the isotropically emitting source. In the case of anisotropic source, the angular size of radiation cannot be arbitrarily large and is limited by $\Delta \theta$. In Fig. A. 1 we show the angular size of the emission region with diameter $D=2 R_{*}=1 \mathrm{Mpc}$. The parameter $\Delta \theta$ has a minimum 1.95 arcmin at redshift $z=1.64$ when the recession velocity equals the speed of light $c$ (Kelner et al. 2011).

\section{References}

Aharonian, F. A. 2002, MNRAS, 332, 215

Aharonian, F. A., Belyanin, A. A., Derishev, E. V., Kocharovsky, V. V., \& Kocharovsky, V. V. 2002, Phys. Rev. D, 66, 023005

Aharonian, F. A., Kelner, S. R., \& Prosekin, A. Y. 2010, Phys. Rev. D, 82, 043002 Ando, S., \& Kusenko, A. 2010, ApJ, 722, L39

Atwood, W. B., Abdo, A. A., Ackermann, M., et al. 2009, ApJ, 697, 1071

Blasi, P., \& Olinto, A. V. 1999, Phys. Rev. D, 59, 023001

Dermer, C. D., Cavadini, M., Razzaque, S., et al. 2011, ApJ, 733, L21

Dolag, K., Grasso, D., Springel, V., \& Tkachev, I. 2005, J. Cosmology Astropart. Phys., 1, 9

Dolag, K., Kachelriess, M., Ostapchenko, S., \& Tomàs, R. 2011, ApJ, 727, L4

Essey, W., Kalashev, O., Kusenko, A., \& Beacom, J. F. 2011, ApJ, 731, 51

Franceschini, A., Rodighiero, G., \& Vaccari, M. 2008, A\&A, 487, 837

Gabici, S. \& Aharonian, F. A. 2005, Phys. Rev. Lett., 95, 251102

Kelner, S. R., Prosekin, A. Y., \& Aharonian, F. A. 2011, Phys. Rev. D, 84, 044016 Kotera, K., Allard, D., \& Lemoine, M. 2011, A\&A, 527, A54

Lehmer, B. D., Brandt, W. N., Alexander, D. M., et al. 2005, ApJS, 161, 21

Neronov, A., \& Vovk, I. 2010, Science, 328, 73

Neronov, A., Semikoz, D. V., Tinyakov, P. G., \& Tkachev, I. I. 2011, A\&A, 526, A90

Romano, P., Campana, S., Mignani, R. P., et al. 2008, A\&A, 488, 1221

Ryu, D., Kang, H., \& Biermann, P. L. 1998, A\&A, 335, 19

Sigl, G., Miniati, F., \& Enßlin, T. A. 2004, Phys. Rev. D, 70, 043007

Tavecchio, F., Ghisellini, G., Foschini, L., et al. 2010, MNRAS, 406, L70

Taylor, A. M., Vovk, I., \& Neronov, A. 2011, A\&A, 529, A144 